\title{
Grace Davie, The Sociology of Religion. A Critical Agenda
}

Second Edition

London, Sage Publications, 2013, 328 p.

\section{Pierre Lassave}

\section{(2) OpenEdition}

Journals

Édition électronique

URL : http://journals.openedition.org/assr/25452

DOI : $10.4000 /$ assr. 25452

ISSN : 1777-5825

Éditeur

Éditions de l'EHESS

Édition imprimée

Date de publication : 30 décembre 2013

Pagination : 176

ISSN : 0335-5985

\section{Référence électronique}

Pierre Lassave, "Grace Davie, The Sociology of Religion. A Critical Agenda », Archives de sciences sociales des religions [En ligne], 164 | 2013, mis en ligne le 20 février 2014, consulté le 21 septembre 2020. URL : http://journals.openedition.org/assr/25452 ; DOI : https://doi.org/10.4000/assr.25452

Ce document a été généré automatiquement le 21 septembre 2020.

(C) Archives de sciences sociales des religions 


\section{Grace Davie, The Sociology of Religion. A Critical Agenda}

Second Edition

London, Sage Publications, 2013, 328 p.

Pierre Lassave

\section{RÉFÉRENCE}

Grace Davie, The Sociology of Religion. A Critical Agenda, Second Edition, London, Sage Publications, 2013, 328 p. 
1 Voici la réédition d'un manuel qui fait déjà partie des classiques dans le monde anglophone de la sociologie des religions. Une reprise de texte de quelques années d'écart avec la première édition (2007) mais qui, tout en gardant l'essentiel de la structure et des références initiales, témoigne de la réflexion continue de l'auteur sur les acquis et les limites du paradigme de la sécularisation et sur les voies plurielles de la modernité religieuse dans le monde.

2 L'ouvrage se divise en deux grandes parties : il retrace d'abord les principales constructions théoriques en s'attachant à indiquer leur contexte d'émergence et à préciser leurs derniers développements; il aborde ensuite une série de thèmes clés qui vont $\mathrm{du}$ devenir des Églises

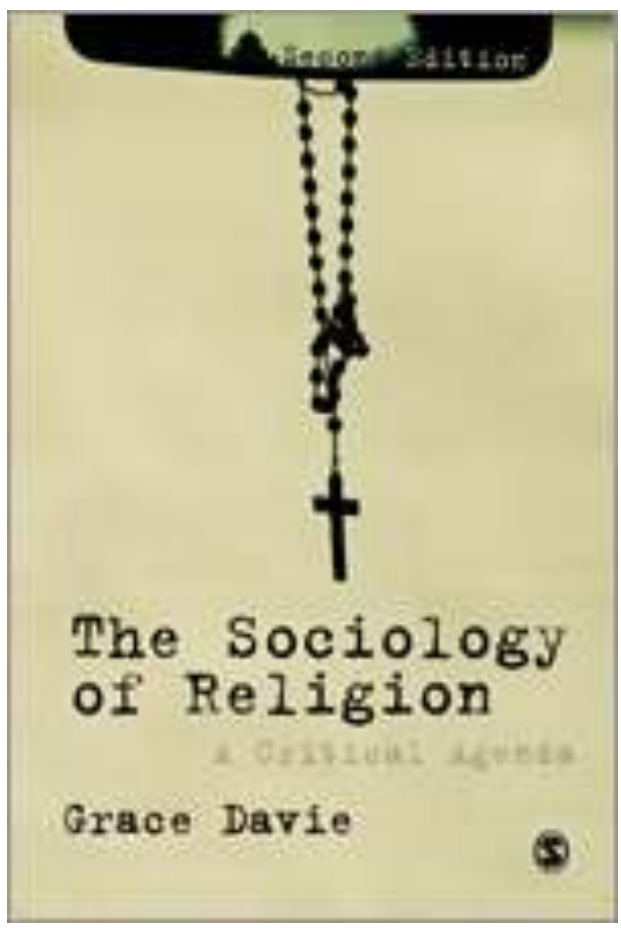
historiques aux polarités variées de la religiosité ordinaire. Se définissant comme « agenda critique », le manuel présente les questions majeures que se pose la discipline académique confrontée à un objet, les faits dits religieux, en pleine mouvance à l'échelle des divers continents. Peut-on ainsi parler de retour du religieux dans le monde laissant ainsi entendre qu'il aurait un temps disparu? Comment comprendre que les questions religieuses soient renvoyées à la conscience privée de chacun alors que l'espace public bruit des affaires invoquées au nom d'un dieu absolu? Comment expliquer que le culte rendu au même dieu ait engendré ici son oubli et ailleurs des regains de ferveur? Quel effet peut produire un monde où les cultures et les traditions longtemps séparées se rapprochent et s'interpénètrent selon un rythme toujours plus intense? La conception occidentale de la modernité issue de la Réforme, de l'humanisme renaissant, de la philosophie rationnelle des Lumières, de l'industrialisation et de l'urbanisation ne s'en trouve-t-elle pas transformée?

Tirant le bilan personnel et collectif d'une vingtaine d'années d'enquêtes et de réflexions sur ces questions, Grace Davie précise d'emblée que ce manuel, principalement destiné au public universitaire, ne traite pas directement des religions dans le monde mais de la manière dont la sociologie les aborde, les décrit, les conceptualise. À cet égard, l'idée que les croyances et les pratiques se référant à des choses sacrées sont menacées d'obsolescence est consubstantielle de la naissance de cette discipline centrale de la connaissance des sociétés par elles-mêmes. D'où sans doute l'influence quasi naturelle du paradigme de la sécularisation sur la façon de saisir les faits religieux, ne serait-ce qu'en les séparant des autres activités sociales, différenciation issue de l'histoire de la modernité occidentale que l'on ne retrouve pas à l'examen d'autres contextes culturels. D'où également, et notamment en regard d'une perspective anthropologique, la relative fragilité épistémique de l'objet religieux pour la sociologie. James Beckford, cité par l'auteur, s'explique ainsi «l'insularité » de la sociologie des religions au sein des sciences humaines. 
4 Mais à l'instar d'autres handbooks parus dans la dernière décennie, cet agenda critique atteste de la vitalité de la "sous-discipline » en question en faisant le lien entre une série de faits qui occupent l'actualité et des explications conscientes de leurs limites et de leurs causes. Plus encore, l'histoire même de la sociologie a partie liée avec le religieux non seulement parce qu'elle s'est construite en l'objectivant à la manière d'une chose sociale parmi d'autres, mais aussi parce qu'elle a placé cette dernière au centre de la logique d'engendrement du lien social tant au niveau des catégories de pensée que des schèmes d'action. On reconnaît là l'influence de Durkheim, premier sociologue à avoir tenté une définition positive de la religion au début du siècle dernier en mettant en avant la puissance d'association propre à l'interdit tant désiré qui préside à tout classement et regroupement social. Outre Durkheim, le manuel s'en tient à Marx, Weber et Simmel comme pères fondateurs. Marx pour avoir situé le sentiment religieux comme illusion sociale historiquement nécessaire, véritable "soupir de la créature opprimée " par les temps de domination d'une classe sur l'autre, mais qui devrait perdre sa raison d'être lorsque les classes auront disparues. Simmel pour avoir plus prudemment décrit les mécanismes modernes de l'individualisation de cette illusion. Et surtout, entre les deux, Weber pour avoir fourni au sociologue d'aujourd'hui les meilleurs outils d'analyse de ce qui se joue autour de la scène religieuse, tant en terme de formes de légitimation collective qu'en termes d'investissements personnels et d'engagements politiques. De là, diverses filiations intellectuelles qui vont se déployer jusqu'à la fin du $\mathrm{xx}^{\mathrm{e}}$ siècle de part et d'autre de l'Atlantique, les unes plus centrées sur ce que les engagements religieux font à la société, les autres sur ce qui les qualifie en substance.

5 Après la Seconde Guerre mondiale, les sociologues américains semblent avoir la main sur le devenir de la sous-discipline en associant la sécularisation du monde au stade ultime de la modernité. En même temps, leurs enquêtes dans le Nouveau Monde montrent que l'urbanisation des mœurs et des idées s'accompagne d'une vitalité religieuse remarquablement plus grande que dans la vieille Europe et ce quelle que soit la séparation formelle entre les Églises et l'État. Les signes de l'émancipation hors des cadres de pensée et d'action forgés par des millénaires d'assujettissement semblent par contraste nettement plus avancés dans un pays de contrat de laïcité comme la France. Mais les forces hétérogènes qui travaillent une société ouverte aux flux du monde rendent les différences nationales à leur devenir incertain. Des revendications mettant en avant une tradition parmi d'autres, comme le port du voile par certaines collégiennes nées dans des familles qui se reconnaissent dans la religion musulmane, suffisent à ébranler le consensus républicain qui veut que tout signe religieux soit abandonné à l'entrée du collège.

6 Pédagogique, le manuel n'en reprend pas moins les multiples acceptions théoriques de la sécularisation, par exemple, la différenciation croissante des activités sociales, la pluralisation des «structures de plausibilité » (Peter Berger), l'individualisation des rapports aux valeurs (Steve Bruce), l'injonction de plus en plus insistante à l'identification catégorielle de soi ("l'impératif hérétique » selon Berger). Il montre également comment ce paradigme envahissant (pervasive) faillit à son rôle explicatif lorsque certains, ou les mêmes qui l'ont construit, le remettent en cause dans un mouvement de balancier trop simple pour être vrai (desecularization). Les comparaisons entre les continents confirment à tout le moins des voies de sécularisation de plus en plus différentes entre elles. Le type européen, marqué par des Églises à vocation 
universelle et aux racines étatiques, contraste ainsi nettement avec la mosaïque américaine de confessions multiples assemblées autour d'un idéal national commun de « religion civile » (Robert Bellah).

7 Depuis une vingtaine d'années fleurissent sur les décombres des théories de la sécularisation diverses explications de la présence renouvelée des croyances et des appartenances dans le moment contemporain. L'auteur s'appuie à de nombreuses reprises sur l'idée de pluralisation des «modernités religieuses" développée par Schmuel Eisenstadt pour présenter les modèles à causalité multiple qui s'y rattachent. On rencontre ainsi du côté américain une application délibérée des théories du choix rationnel au «marché religieux » (Stephen Warner). Sur un continent où la plupart des habitants croient au divin et souscrivent à des communautés religieuses concurrentes, l'orientation de chacun vers telle ou telle Église est alors censée dépendre des avantages qu'elle lui procure par rapport à d'autres (Rodney Stark, William S. Bainbridge). L'offre de services religieux induit la demande de biens de salut (Roger Finke). Mais de même que le paradigme de la sécularisation marque le pas au-delà de l'Europe, la théorie du choix rationnel convient peu à cette dernière où nombre d'églises sont devenues des lieux touristiques. S'intercalant entre les continents, Grace Davie énonce alors sa théorie de moyenne portée qui consiste à marquer un écart entre la croyance et la participation (Believing without belonging) ou à mettre l'accent sur les phénomènes de délégation religieuse (Vicarious religion), croyances latentes qui se révèlent lors d'événements exceptionnels comme ces églises brusquement remplies à l'occasion des funérailles de la princesse Diana. Dans la même veine de dépassement des apories de la sécularisation, l'auteur évoque les thèses de Danièle Hervieu-Léger qui mettent en évidence la dimension mémorielle de toute pratique religieuse, notamment en régime de modernité avancée.

8 Autant de théories, autant de questions de méthode. L'auteur présente ainsi un panorama de problèmes difficiles à résoudre comme celui de la mesure des délégations implicites de culte qui se révèlent $a$ contrario comme dans le cas évoqué des rites de deuil autour de la princesse Diana. Au-delà de l'inévitable débat entre sondages quantitatifs et descriptions ethnographiques, le panorama s'arrête hélas un peu vite sur la question des rapports entre traditions disciplinaires. Certes se confirme le faible poids de la psychologie des religions dans le monde anglophone, hormis l'enclave psychanalytique, ou bien encore l'empiètement croissant de la science politique sur la sociologie des religions. Mais en dehors du fait que l'ethnographie peut servir de descripteur sociologique, peu est dit des rapports entre anthropologie, histoire et sociologie des religions. Trois spécialités disciplinaires qui se disputent pourtant depuis plus d'un siècle la connaissance empirique des phénomènes religieux. On peut se demander d'ailleurs si l'optique fonctionnaliste qui prévaut dans les théories de la modernité religieuse n'explique pas le peu de cas qui est ici fait de la nature ou de la substance même des engagements taxés de religieux, tous aspects qui mobilisent plutôt le philosophe, l'historien et l'anthropologue. Rien d'étonnant d'ailleurs à ce qu'on ne trouve pas plus de développement sur les interactions éventuelles, ne fût-ce qu'historiques, entre sociologie et théologie. Sans parler de la réflexion absente ici, mais qui occupe plutôt les anthropologues sur leur rapport de familiarité ou de distance avec l'objet religieux, qu'il s'agisse par exemple de faire le partage entre " athéisme méthodologique » et « agnosticisme méthodologique ». 
9 Après ces quelques considérations de méthode qui concluent la partie théorique suit une série d'approches thématiques qui se recoupent quelque peu avec les modèles explicatifs déjà présentés : religions historiques, minorités actives, fondamentalismes, globalisation, religion au quotidien. Le cas de la montée en régime des groupements et réseaux radicaux est typique de l'approche macrosociologique qui imprègne le manuel. $\mathrm{Au}$-delà de la genèse de ces mouvements de réaction antimoderniste et de la description événementielle de ses manifestations les plus violentes, l'auteur insiste ainsi sur les mécanismes de recharge de certitude qui les fait revivre dans un monde déserté par les grands récits qui donnent sens à la vie humaine et au-delà. Les effets de stigmatisations réciproques que certains analystes des interactions symboliques proches de la Labelling theory sont ici absents du tableau. De même, à propos de la religion vécue au jour le jour, le lecteur ne rencontrera que le jeu des institutions publiques et confessionnelles qui prennent diversement en charge selon les pays les questions de la vie, de la maladie et de la mort. L'allongement de la durée de vie, l'émancipation des femmes, la recomposition des familles ou les questions de la procréation assistée tiennent ainsi lieu de variables d'observation des changements qui affectent les institutions. Mais on peinera à trouver quelques considérations sur la fluidité des croyances de toutes natures pour y déceler la place de ce qu'on appelle le religieux. Point de considération sur les liens éventuels entre croire, vouloir ou devoir. L'optique sociopolitique dominante de cette sociologie des religions anglophone nous vaut cependant d'intéressantes pages de synthèse sur les agencements religieux de l'histoire contemporaine. Il en va ainsi de la reconstitution de la figure symboliquement protéiforme du pape Jean-Paul II, tout à la fois renverseur de murs comme celui de Berlin, apôtre des opprimés et gendarme des mœurs. Il en est également des jeux complexes entre le revivalisme islamique et l'état de laïcité dans des pays émergents comme la Turquie ou l'Indonésie.

10 Attentive à la diversité des situations, l'auteur récapitule pour finir les défis que l'époque contemporaine lance à sa spécialité. Comment ainsi rendre compte de la mobilité des cadres de références qui affectent tant les croyances et les Églises que le regard sociologique posé sur ces dernières ? Comment distinguer les effets réciproques du jeu entre le centre et les marges des institutions en question? Si l'on convient que cette mobilité s'est accélérée au fil d'un temps marqué par des événements clés comme la révolution iranienne de 1979, la chute du mur de Berlin de 1989 ou la destruction des tours de New York en 2001, comment ne pas s'interroger sur le décalage avec la réalité qu'induit le paradigme trop longtemps dominant de la sécularisation? La sociologie des religions se trouve ainsi prise dans une sorte de course-poursuite avec le réel (l'auteur utilise la métaphore ludique du Leapfrog Que L'on Peut Traduire Par «Jeu A SauteMouton ») Qui En Fait Tout L'intérêt. Le Savoir Avance Ainsi En Sautant D'un Paradigme A L'autre Comme L'a Déjà Montré Thomas S. Kuhn. L'idée De «Modernité Religieuse Multiple » Prétend Ainsi Détrôner Celle De La Sécularisation Du Monde. Les Théories A Moyenne Portée, Telles Celles Evoquées Sur Les Délégations Vicariales Ou Sur L'inscription Des Identités Dans Une Lignée Mémorielle, Indiquent Que Les Jeux Sont Ouverts. 